NBER WORKING PAPER SERIES

COALITION-PROOF TRADE AND THE FRIEDMAN RULE IN THE LAGOS-WRIGHT MODEL

Tai-wei Hu

John Kennan

Neil Wallace

Working Paper 13310

http://www.nber.org/papers/w13310

NATIONAL BUREAU OF ECONOMIC RESEARCH

1050 Massachusetts Avenue

Cambridge, MA 02138

August 2007

This research was supported by grants from the National Science Foundation. An earlier version circulated under the title "Pairwise-core monetary trade in the Lagos-Wright model." The views expressed herein are those of the author(s) and do not necessarily reflect the views of the National Bureau of Economic Research.

(C) 2007 by Tai-wei Hu, John Kennan, and Neil Wallace. All rights reserved. Short sections of text, not to exceed two paragraphs, may be quoted without explicit permission provided that full credit, including $\odot$ notice, is given to the source. 
Coalition-Proof Trade and the Friedman Rule in the Lagos-Wright Model

Tai-wei Hu, John Kennan, and Neil Wallace

NBER Working Paper No. 13310

August 2007, Revised October 2008

JEL No. E40

\begin{abstract}
$\underline{\text { ABSTRACT }}$
The Lagos-Wright model -- a monetary model in which pairwise meetings alternate in time with a centralized meeting -- has been extensively analyzed, but always using particular trading protocols. Here, trading protocols are replaced by two alternative notions of implementability: one that allows only individual defections and one that also allows cooperative defections in meetings. It is shown that the first-best allocation is implementable under the stricter notion with- out taxation if people are sufficiently patient. And, if people are free to skip the centralized meeting, then lump-sum taxation used to pay interest on money does not enlarge the set of implementable allocations.
\end{abstract}

Tai-wei Hu

Department of Economics

The Pennsylvania State University

608 Kern Graduate Building

University Park, PA 16802-3306

tuh129@psu.edu

John Kennan

Department of Economics

University of Wisconsin

1180 Observatory Drive

Madison, WI 53706

and NBER

jkennan@ssc.wisc.edu

\author{
Neil Wallace \\ Department of Economics \\ The Pennsylvania State University \\ 612 Kern Graduate Building \\ University Park, PA 16802-3306 \\ neilw@psu.edu
}




\title{
Coalition-Proof Trade and the Friedman Rule in the Lagos-Wright Model*
}

\author{
Tai-wei Hu† John Kennan $\ddagger$ and Neil Wallace ${ }^{\S}$
}

September 18, 2008

\begin{abstract}
The Lagos-Wright model-a monetary model in which pairwise meetings alternate in time with a centralized meeting - has been extensively analyzed, but always using particular trading protocols. Here, trading protocols are replaced by two alternative notions of implementability: one that allows only individual defections and one that also allows cooperative defections in meetings. It is shown that the first-best allocation is implementable under the stricter notion without taxation if people are sufficiently patient. And, if people are free to skip the centralized meeting, then lump-sum taxation used to pay interest on money does not enlarge the set of implementable allocations. (100 words)
\end{abstract}

Keywords: matching model, coalition-proof, optima

JEL classification: E-40.

\section{Introduction}

Models in which people meet in pairs are common in monetary economics and labor economics. In monetary economics, absence-of-double-coincidence

*This research was supported by grants from the National Science Foundation. An earlier version circulated under the title "Pairwise-core monetary trade in the LagosWright model."

$\dagger$ The Pennsylvania State University: <tuh129@psu.edu>.

†niversity of Wisconsin, Madison and NBER $<$ jkennan@ssc.wisc.edu $>$

$\S$ The Pennsylvania State University: < neilw@psu.edu>. 
situations have almost always been described in terms of such meetings. In addition, models with pairwise meetings have been useful in applications. ${ }^{1}$ However, such models give rise to an old question: how is trade determined in bilateral-monopoly situations? By far the most common approach is to assume one or several trading protocols; examples are alternating offers, take-it-or-leave-it offers, bargaining according to Nash, and posted prices. Another approach, a kind of mechanism-design approach, explores all implementable outcomes. Here, we apply the implementability approach to the Lagos-Wright (2005) model, a model in which pairwise meetings alternate in time with a centralized meeting in which there is competitive trade.

Lagos-Wright is a convenient model in which to emphasize the differences between the consequences of our implementability approach and the trading-protocol approach. First, it is known that the model's implications for optima - and, more generally, for the welfare costs of inflation - are sensitive to the trading protocol (see Lagos and Rocheteau 2005, Lagos and Wright 2005, and Rocheteau and Wright 2005). For example, Lagos and Wright, using a parameterized version of their model, find that a $10 \%$ inflation is equivalent to a $1.4 \%$ reduction in consumption under buyer take-itor-leave-it offers, as compared with a $3.2 \%$ reduction under Nash bargaining. We provide a more robust analysis by, in effect, searching over all trading protocols that satisfy some properties. Second, the model has a crucial simplifying assumption: quasi-linear preferences in the centralized meeting. That assumption, which is consistent with a degenerate steady-state distribution of money and accounts for the model's popularity, allows us to say a lot about the set of implementable outcomes.

We apply two notions of implementability to the model. One notion, called individually-rational (IR) implementability, requires only that trades in pairwise meetings be immune to individual defection; the other, called coalition-proof (CP) implementability, also requires that those trades be immune to cooperative defection by the pair in a meeting. ${ }^{2}$ For both notions, we maintain the competitive trade in the centralized meeting assumed in Lagos-Wright. Such trade is consistent with CP implementability because the outcome of competitive trade is the core for such meetings. As between

\footnotetext{
${ }^{1}$ For example, pairwise meetings are used in a crucial way to generate float in Wallace and Zhu (2007).

${ }^{2}$ Earlier applications of IR implementability in monetary models include Kocherlakota (1998). One application of CP implementability in monetary models is Deviatov (2006), who studies optimal inflation numerically.
} 
the two notions, we prefer CP implementability because it is consistent with exhaustion of the gains from trade in meetings. We follow existing work by studying the model without and with lump-taxes levied in the centralized meeting and used to finance interest on money. The version with taxes can be used to study the role of the Friedman rule and the welfare costs of inflation.

According to all the previously studied trading protocols in the LagosWright model, the first-best allocation is not achievable without the use of lump-sum taxes. In contrast, we show that the first-best is CP implementable

without taxes when people are sufficiently patient. Previous expositions of the model also show that the first-best is achievable if buyers make take-itor-leave-it offers and if there is lump-sum-tax financed payment of interest on money at the Friedman-rule rate. We show that if preferences in the centralized meeting are linear, rather than quasi-linear, and people are free to skip the centralized meeting - an implication of no-commitment by individuals that has been ignored - then such a policy does not help; it does not enlarge even the set of IR implementable allocations. (Although we use linearity throughout, this is the only result that depends on it.) Finally, as noted above, previous studies show that the welfare cost of inflation varies greatly with the trading protocol. We characterize the sets of IR and CP implementable allocations taking as given the inflation rate. If we measure the welfare cost of inflation by choosing the best $\mathrm{CP}$ implementable allocation subject to a given inflation rate, then we find an associated welfare cost, measured relative to the first best, that is smaller than that found for any given trading protocol. Indeed, for the Lagos-Wright parameterized version, inflation less than about $16 \%$ per year is costless.

\section{The environment}

Time is discrete, there are two stages at each date, preferences are additively separable over dates and stages, and there is a nonatomic unit measure of people who maximize expected discounted utility with discount factor $\delta \in$ $(0,1)$. The first stage has pairwise meetings and the second stage has a centralized meeting. Just prior to the first stage, a person looks forward to be being a buyer who meets a seller with probability $\frac{1}{N}$, looks forward to being a seller who meets a buyer with probability $\frac{1}{N}$, and looks forward to no pairwise meeting with probability $1-\frac{2}{N}$, where $N \geq 2$. The stage-1 
utility of someone who becomes a seller and produces $y \in \mathbb{R}_{+}$is $-c(y)$, while that of someone who becomes a buyer and who consumes $y$ is $u(y)$, where $c(0)=u(0)=0, c$ and $u$ are strictly increasing and continuous with $c$ convex and $u$ concave, and $u-c$ is strictly concave. ${ }^{3}$ Moreover, there exists $y>0$ such that $c(y)=u(y)$. There are special preferences for stage 2: the utility of consuming $z$ amount of the stage- 2 good is $z$. As in Lagos and Wright (2005), $z<0$ is interpreted as production. ${ }^{4}$

All goods are perishable (both across stages and time), people cannot commit to future actions, and there is no monitoring (histories are private information)-assumptions that serve to make money essential. Money is divisible, in fixed supply, and the per capita amount is normalized to be 1 . Finally, people can hide money and participation in the centralized meeting is voluntary, voluntariness that matters only when there are lump-sum taxes.

\section{$3 \quad$ Stationary and symmetric allocations}

All of our results are about allocations in which consumption and production are stationary and symmetric in the following way. An allocation is a pair $(y, z) \in \mathbb{R}_{+}^{2}$, where $y$ is stage- 1 production and consumption in any buyerseller meeting and $z$ is stage- 2 production (consumption) in the centralized meeting of any person who consumed (produced) y at stage 1. Associated with such $(y, z)$ is zero production and consumption at both stages by those who didn't meet anyone at stage 1 .

We describe the set $(y, z)$ that is IR implementable and the subset that is CP implementable. When judging the welfare of $(y, z)$, our criterion is the payoff implied by $(y, z)$ prior to pairwise meetings; namely, $h(y) \equiv \frac{u(y)-c(y)}{N(1-\delta)}$. (Because a person is as likely to produce $z$ as to consume $z$ and because stage 2 utility is linear, the magnitude of $z$ does not appear in this expression.)

The assumed strict concavity of $u-c$ implies that $\arg \max [u(y)-c(y)]$, denoted $y^{*}$, is unique, and that $h(y)$ is strictly increasing for $y \in\left[0, y^{*}\right]$ and strictly decreasing for $y \in\left[y^{*}, \infty\right)$. Our assumptions also imply that $y^{*}>0$.

\footnotetext{
${ }^{3}$ The assumption $c(0)=u(0)=0$ is without loss of generality. If it does not hold, then in all the expressions that follow we replace $u(y)$ by $u(y)-u(0)$ and $c(y)$ by $c(y)-c(0)$.

${ }^{4}$ In Lagos and Wright (2005), the authors assume quasi-linearity and a net gain from producing $z$ and consuming $z$ for some $z$. As noted above, only one of our results depends on our linearity assumption, as opposed to their quasi-linearity assumption.
} 


\section{Implementable allocations: zero taxes}

Both of our notions of implementability are weak in the sense that when we say that an allocation is implementable we mean that there exists an equilibrium with that allocation as an outcome. We will not be demonstrating that any equilibrium gives that outcome. In addition, the equilibrium notion we use relies on anonymity. That is, each person in a pairwise meeting evaluates the consequences of current actions taking as given that the people each will meet in the future will have money holdings implied by equilibrium play.

We next describe two games: one for each notion of implementability. Each game is defined relative to a (planner) proposal. We use $\mathbb{M} \subset \mathbb{R}_{+}$to denote the set in which money holdings or money transfers reside; $\mathbb{Y} \subset \mathbb{R}_{+}$ to denote the set in which stage 1 output resides, and $\mathbb{Z} \subset \mathbb{R}$ to denote the set in which stage- 2 consumption resides.

Definition 1 A proposal consists of three objects: (i) an initial distribution of money; (ii) a function that describes trades in stage-1 meetings, $g: \mathbb{M}^{2} \rightarrow$ $\mathbb{Y} \times \mathbb{M}$, where the domain is announced money holdings of the buyer and the seller, respectively, and the range is output (produced by the seller and consumed by the buyer) and the transfer of money from the buyer to the seller; (iii) a price of money, denoted $p \in \mathbb{R}_{+}$, for the stage 2 centralized meeting.

Notice that we are limiting consideration to $g$ and $p$ that are constant over time.

Both games have each agent choosing a budget-feasible trade at the price of money $p$ at stage 2 . To deal with the fact that an arbitrary profile of individual budget-feasible choices is not feasible, we allow the planner to satisfy stage- 2 excess demands by giving the planner unlimited access to money and access to the same stage- 2 linear technology that agents have. ${ }^{5}$ Now we describe the sequence of actions in stage 1 meetings, a sequence that differs for the two games.

\footnotetext{
${ }^{5}$ For this setting, it would possible to replace stage-2 competitive trade by a ShapleyShubik trading post, Cournot-type game (see Shubik 1973). Because there is a nonatomic measure of agents, one equilibrium of that game would coincide with competitive equilibrium. (The existence of other equilibria - in particular, a no-trade equilibrium - would not be a concern because our notions of implementability are weak.) If we did use the trading-post formulation, then we would have feasibility for any agent choices at stage 2 without involving the planner.
} 
The IR-game. First, the buyer and seller simultaneously announce their money holdings. Second, they simultaneously choose from $\{y e s, n o\}$. If both say yes, then the trade given by the proposal is carried out; otherwise the meeting is autarkic.

Obviously, the second step insures that any trade that occurs is individually rational.

The CP-game. First, the buyer and seller simultaneously announce their money holdings. Second, they simultaneously choose from $\{y e s$, no $\}$. If both say yes, then they go to the next step; otherwise the meeting is autarkic. Third, the buyer announces a trade and then the seller announces from $\{y e s, n o\}$. If the seller announces yes, then the buyer's proposed trade is carried out; otherwise, the trade in the (planner's) proposal is carried out. ${ }^{6}$

The second step in the $\mathrm{CP}$ game insures that each person has the option of autarky. If the third step is reached, then either the planner's proposal is carried out or a trade that Pareto dominates it is carried out. Therefore, if the planner's proposal is coalition proof for the pair, then it is carried out. In that case, there is no benefit to the buyer in the third step from proposing. If the planner's proposal is not coalition proof, then there is some benefit, but it is constrained by the planner's proposal.

We focus on simple strategies in these games.

Definition 2 A strategy in the IR game consists of three functions, denoted $\left(s_{b}^{t}, s_{s}^{t}, s_{c}^{t}\right)$, the first two pertaining to stage 1 and the third to stage 2: $s_{b}^{t}=$ $\left(s_{b 1}^{t}, s_{b 2}^{t}\right)$, where $s_{b 1}^{t}: \mathbb{M} \rightarrow \mathbb{M}$ is the buyer's announced money holdings and $s_{b 2}^{t}: \mathbb{M} \times \mathbb{M} \rightarrow\{y e s, n o\}$, where the first set in the domain is the buyer's money holdings and the second is the seller's announced money holding. ${ }^{7}$ $s_{s}^{t}=\left(s_{s 1}^{t}, s_{s 2}^{t}\right)$, defined analogously for the seller; and $s_{c}^{t}: \mathbb{M} \rightarrow \mathbb{Z}$ is the choice of consumption in the centralized meeting (the domain is money holdings at the start of stage 2).

${ }^{6}$ This CP game specification is borrowed from Zhu (2008). Many alternatives would imply our results. For example, in the last step, the roles of the buyer and seller could be reversed or there could be random determination of who makes the trade proposal.

${ }^{7} \mathrm{~A}$ formal definition of strategies should add the buyer's announced money holding to the domain here and in the definition for the CP game that follows. We omit it because including it would complicate the notation and would not affect the results. 
Definition 3 A strategy in the CP game consists of three functions, again denoted $\left(s_{b}^{t}, s_{s}^{t}, s_{c}^{t}\right): s_{b}^{t}=\left(s_{b 1}^{t}, s_{b 2}^{t}, s_{b 3}^{t}\right)$, where the first two components have domains and ranges as in the IR game and $s_{b 3}^{t}: \mathbb{M} \times \mathbb{M} \rightarrow \mathbb{Y} \times \mathbb{M}$ where the first set in the domain is the buyer's money holdings and the second is the seller's announced money holding and the range is the set of buyer proposed trades; $s_{s}^{t}=\left(s_{s 1}^{t}, s_{s 2}^{t}, s_{s 3}^{t}\right)$, where the first two components have domains and ranges as in the IR game and where $s_{s 3}^{t}:(\mathbb{M} \times \mathbb{M}) \times(\mathbb{Y} \times \mathbb{M}) \rightarrow\{$ yes, no $\}$ $i s$ the seller's response to the buyer's proposed trade; and $s_{c}^{t}$ defined as in the IR game.

Implicit in the above is that a triple $\left(s_{b}^{t}, s_{s}^{t}, s_{c}^{t}\right)$ is a strategy only if it satisfies the obvious feasibility constraints; in particular, whether making an announcement, a trade proposal, or a trade, agents cannot overstate money holdings. These strategies are simple in that they are not contingent on the agent's past private history. For the moment, they depend on the date because we are not building into the definition of equilibrium an unchanged distribution of money holdings. Notice that the function $s_{c}^{t}$ also determines an end-of-date money holding because the choice satisfies the person's budget at equality. Therefore, given a (planner) proposal and a strategy $\left\{s_{b}^{t}, s_{s}^{t}, s_{c}^{t}\right\}_{t=0}^{\infty}$, there are implied money distributions at each date, $\varphi_{p}^{t}$ before pairwise meetings and $\varphi_{c}^{t}$ before the centralized meeting, and implied value functions, $w_{p}^{t}: \mathbb{M} \rightarrow \mathbb{R}$ before pairwise meetings and $w_{c}^{t}: \mathbb{M} \rightarrow \mathbb{R}$ before the centralized meeting.

Finally, we have to introduce buyer and seller beliefs. A belief is $\gamma^{t}=$ $\left(\gamma_{b}^{t}, \gamma_{s}^{t}\right)$, where each component maps $\mathbb{M}$ (partner's announced money holding) to $\Delta(\mathbb{M})$, a distribution over partner's money holding. In the IR-game, $\gamma^{t}$ does not matter because players are concerned only with the planner's proposed trade which is completely determined by the reported money holdings. In the CP-game, types also matter because they help determine the alternative trades that could be carried out.

In terms of the above notation, we have the following definition of an equilibrium.

Definition 4 An equilibrium of the IR (CP) game is a sequence $\left\{\left(s_{b}^{t}, s_{s}^{t}, s_{c}^{t}\right), \quad\left(\varphi_{p}^{t+1} \varphi_{c}^{t}\right), \gamma^{t}\right\}_{t=0}^{\infty}$ that satisfies (i) $\left(s_{b}^{t}, s_{s}^{t}\right)$ is optimal given $w_{c}^{t}$ and $\gamma^{t}$, and $s_{c}^{t}$ is optimal given $w_{p}^{t+1}$; (ii) $\gamma^{t}$ is implied by $\varphi_{p}^{t}$ and $\left(s_{b}^{t}, s_{s}^{t}\right)$ via Bayes rule whenever possible; and (iii) $\int s_{c}^{t} d \varphi_{c}^{t}=0$. 
In this definition, we take for granted that the value functions and the distributions are those implied by the initial condition and the strategies. As noted above, equilibrium is defined relative to a planner's proposal which includes $\varphi_{p}^{0}$, the initial condition. Condition (i) is a Nash-like feature, because checking whether a sequence satisfies it involves checking whether it holds for the value functions implied by the sequence. As written, condition (i) appeals to the one-date deviation principle which, as pointed out below, applies. Condition (ii) is standard and condition (iii) is feasibility at stage 2 without planner participation.

Throughout we work with a special kind of equilibrium.

Definition 5 A simple equilibrium is an equilibrium in which (i) the proposal has a degenerate initial distribution of money (each person has 1 unit); (ii) the strategy and belief sequences are constant sequences and imply $\varphi_{p}^{t}=\varphi_{p}^{0}$; (iii) everyone is truthful, the planner's proposed trade is carried out, and yes is always played.

Now we can define implementability for the stationary and symmetric allocations introduced in the last section.

Definition 6 The allocation $(y, z)$ is IR (CP) implementable if there exists a simple equilibrium of the IR (CP) game whose outcome is consistent with $(y, z)$.

An obvious consequence of this definition is that IR implementability is necessary for $\mathrm{CP}$ implementability. We also have the following necessary condition for IR implementability.

Lemma 1 If $(y, z)$ is IR implementable, then the planner's proposal satisfies the following conditions: $\varphi_{p}^{0}$ is degenerate, $g(1,1)=(y, 1)$, and $p=z$. Moreover, the implied value functions satisfy

$$
w_{p}^{t}(1)=h(y) \text { and } w_{c}^{t}(m)=z(m-1)+\delta w_{p}^{t}(1) \text { for } m \in\{0,1,2\} .
$$

Proof. By definition 6, there exists a simple equilibrium whose outcome is consistent with $(y, z)$. If the corresponding proposal does not satisfy $g(1,1)=(y, 1)$, then it must satisfy $g(1,1)=(y, x)$, with $x<1$. But any such trade in a simple equilibrium implies that people enter stage 1 with more money than they will spend, even if they are buyers. This violates optimal 
stage-2 choice at the previous date because linearity of stage- 2 utility and discounting implies that any such person is better off entering stage 1 with only $x$ amount of money and restoring money holdings at the next stage 2 . With $g(1,1)=(y, 1)$, only $p=z$ is consistent with $(y, z)$ and with persistence of the degenerate distribution of money. The value function claims are obvious.

Our most important results demonstrate implementability of some $(y, z)$. For such sufficiency-like results, the restriction to simple equilibria is without loss of generality. The crucial part of the proof for each such result is the construction of the (planner's) proposal. The on-equilibrium parts of the proposal are in lemma 1. That is, the seller produces $y$ and the buyer transfers all money held. And at $p=z$, agents make choices that restore the degenerate distribution and, as a consequence, are feasible. The main effort in our sufficiency proofs is devoted to constructing $g(\cdot, 1)$ and $g(1, \cdot)$, the off-equilibrium trades. For a simple equilibrium, we do not have to define $g$ on other parts of the domain. And for a simple equilibrium, beliefs are easy to specify. If a money holding equal to unity is announced, then Bayes rule applies and implies that the announcement is treated as truthful; if a money holding different from unity is announced, then any belief can be specified because Bayes rule does not apply. The main idea behind the construction of $g(\cdot, 1)$ is to punish the buyer with off-equilibrium money holdings.

Some of our results demonstrate that some $(y, z)$ are not implementable. For such necessity-type results, the restriction to simple equilibria may have bite. That is, for such results, we establish only that there is no simple equilibrium with an outcome consistent with $(y, z)$. And we do that by describing some feasible defections from a simple equilibrium.

\section{Results: zero taxes}

Most of our results can be stated in terms of the set,

$$
V=\left\{(y, z) \in \mathbb{R}_{+}^{2}: c(y) \leq z \leq R u(y)\right\}
$$

where

$$
R=\frac{\delta}{N(1-\delta)+\delta}
$$


Because welfare depends only on $y$, it is useful to describe the projection of $V$ on $y$; namely, the interval $\left[0, y_{\max }\right]$, where $y_{\max }$ is the unique positive solution for $y$ to $c(y)=R u(y)$. (If $y$ is such that $c(y) \leq R u(y)$, then $[c(y), R u(y)]$ is not empty and $(y, z)$ with $z \in[c(y), R u(y)]$ is in the set $V$. And if $(y, z) \in V$, then $y \in\left[0, y_{\max }\right]$.) Moreover, the optimum in $\left[0, y_{\max }\right]$ namely, $\arg \max _{y \in\left[0, y_{\max }\right]} h(y)$-is $\min \left\{y_{\max }, y^{*}\right\} .{ }^{8}$ And, because $R \rightarrow 1$ as $\delta \rightarrow 1$, [0, $\left.y_{\max }\right]$ includes $y^{*}$, the first best, for all sufficiently high $\delta$-all $\delta \geq \delta^{*}$, where $\delta^{*}$ satisfies $c\left(y^{*}\right)=\left(\frac{\delta^{*}}{N\left(1-\delta^{*}\right)+\delta^{*}}\right) u\left(y^{*}\right)$.

We start by characterizing the set of IR implementable allocations.

Proposition 1 There exists z such that $(y, z)$ is IR implementable if and only if $y \in\left[0, y_{\max }\right]$.

Proof. Necessity. At stage 1, defection to no-trade by a seller with 1 unit of money assures a payoff no less than $w_{c}(1)$, while following $g(1,1)=$ $(y, 1)$ gives the seller $-c(y)+w_{c}(2)$. Thus, it must be the case that

$$
c(y) \leq w_{c}(2)-w_{c}(1)=z,
$$

where the equality follows from (1) (see lemma 1). This is the first inequality that defines $V$.

Next, consider an agent who enters stage 2 with 0 money. By lemma 1 , this agent's payoff is $-z+\delta w_{p}(1)$. However, it is feasible for this agent to produce 0 at stage 2 and resume feasible equilibrium actions starting at the next date. This possibility and IR implementability give the inequality

$$
-z+\delta w_{p}(1) \geq-\frac{\delta c(y)}{N}-\frac{(N-1) \delta z}{N}+\delta^{2} w_{p}(1)
$$

where the righthand side is the payoff from the above defection. But, by lemma 1 , this inequality is equivalent to

$$
\delta(1-\delta) h(y) \geq z\left(1-\delta \frac{N-1}{N}\right)-\frac{\delta c(y)}{N} .
$$

Using the definition of $h(y)$, this is easily seen to be the second inequality that defines $V$.

\footnotetext{
${ }^{8}$ The interval $\left[0, y_{\max }\right]$ is the set that can be implemented with perfect monitoring (with a defector punished by permanent autarky) in a version of the model in which stage 2 does not exist.
} 
Sufficiency. Our candidate for completion of the planner's proposal is

$$
g(1, m)=(y, 1) \text { for all } m \text { and } g(m, 1)=\left\{\begin{array}{c}
(y, 1) \text { if } m \geq 1 \\
(0,0) \text { if } m<1
\end{array},\right.
$$

where, recall, the first argument of $g$ is the buyer's announced money holding and the second is the seller's. (Notice that a buyer with less than 1 unit is punished by no trade.) We also propose that an equilibrium strategy has $s_{c}(m)$ (consumption at stage 2) such that each person, with arbitrary $m$, leaves stage 2 with 1 unit of money.

If the above trades are carried out, then the initial degenerate distribution persists and the value functions are

$$
w_{c}(m)=z(m-1)+\delta h(y) \text { for all } m
$$

and

$$
w_{p}(m)=\left\{\begin{array}{l}
\frac{-c(y)}{N}+z\left(\frac{m}{N}+\frac{(N-1)(m-1)}{N}\right)+\delta h(y) \text { if } m<1 \\
\frac{u(y)-c(y)}{N}+z\left(\frac{m-2}{N}+\frac{m}{N}+\frac{(N-2)(m-1)}{N}\right)+\delta h(y) \text { if } m \geq 1
\end{array} .\right.
$$

To complete the proof, we have to show that these induce truth-telling and playing yes at stage 1 and induce the proposed stage- 2 strategy. ${ }^{9}$

Given $g$ in (7), truthfulness is a weakly dominant strategy for everyone. Now we show that a buyer with $m \geq 1$ says yes and that any seller says yes. (For buyers with $m<1$, either action implies no trade.) For the former, we need to show that $u(y)+w_{c}(m-1) \geq w_{c}(m)$ or by (8) that $u(y) \geq z$. This follows from the second inequality that defines $V$. For the latter, we need to show that $-c(y)+w_{c}(m+1) \geq w_{c}(m)$, which is just the first inequality that defines $V$.

Now we show that our stage-2 proposed strategy, $s_{c}(m)=z(m-1)$, is optimal for agents. We proceed by considering two exhaustive sets of alternatives.

\footnotetext{
${ }^{9}$ This is where we use the principle of one-period deviations. To invoke it, we have to show that corresponding to any beneficial deviation is a finite-period deviation. This holds provided that period payoffs for a defector not grow too fast. In our case, period payoffs in pairwise meetings are bounded above by $u\left(y_{\max }\right)$. As for period payoffs in the centralized meeting, they can grow at most arithmetically, by $z$ per date. These imply that corresponding to any beneficial deviation is a finite-period deviation. Given the finite-period deviation, the one-period deviation principle follows by backward induction.
} 
Case (i): $s_{c}(m)<z(m-1)$. This implies leaving stage 2 with more than 1 unit of money. However, just as in the proof of lemma 1, this implies carrying some money from the current stage 2 to the next stage 2 , which is not optimal.

Case (ii): $s_{c}(m)>z(m-1)$. This implies leaving stage 2 with less than 1 unit of money, the amount $m-\frac{x}{z}$, where, for simplicity, we denote $s_{c}(m)$ by $x$. Because this amount is less than 1 , the payoff according to (9) is

$$
x+\delta\left[\frac{-c(y)}{N}+z\left(\frac{m-\frac{x}{z}}{N}+\frac{(N-1)\left(m-\frac{x}{z}-1\right)}{N}\right)+\delta h(y)\right] .
$$

This expression is linear in $x$ and increasing. Therefore, consistent with not carrying money that is not spent at stage 1 , this person chooses $x=z m$ (zero money). Then the above expression is

$$
\left.z m+\delta\left[\frac{-c(y)}{N}-z \frac{(N-1)}{N}\right)+\delta h(y)\right] .
$$

We have to show that this is weakly dominated by the payoff from choosing $s_{c}(m)=z(m-1)$, which according to $(8)$ is $z(m-1)+\delta h(y)$. The required inequality is equivalent to

$$
\left.z+\delta\left[\frac{-c(y)}{N}-z \frac{(N-1)}{N}\right)+\delta h(y)\right] \leq \delta h(y)
$$

which, in turn, is equivalent to inequality (6). But, as noted above, inequality (6) is equivalent to the second inequality that defines $V$.

Now we characterize the set of CP implementable allocations, which turns out to be all IR implementable $y$ 's that do not exceed $y^{*}$.

Proposition 2 There exists z such that $(y, z)$ is CP implementable if and only if $y \in\left[0, \min \left\{y_{\max }, y^{*}\right\}\right]$.

Proof. Necessity. If $y_{\max }<y^{*}$, then any $y>y_{\max }$ is not IR implementable and, hence, is not CP implementable. Thus, suppose that $y_{\max } \geq y^{*}$ and suppose, by way of contradiction, that $(y, z)$ with $y>y^{*}$ is CP-implementable. We show that the buyer, instead of proposing $(y, 1)$, proposes a smaller trade and that the seller accepts the buyer's proposal. 
Let $\epsilon>0$ be such that

$$
u(y-\epsilon)-c(y-\epsilon)>u(y)-c(y) .
$$

Such $\epsilon$ exists because $y>y^{*}$. It follows that there exists $\eta>0$ such that

$$
c(y)-c(y-\epsilon)>\eta z>u(y)-u(y-\epsilon) .
$$

Then

$$
\begin{aligned}
u(y-\epsilon)+w_{c}(\eta) & \geq u(y-\epsilon)+z(\eta-1)+\delta w_{p}(1)> \\
u(y)-z+\delta w_{p}(1) & =u(y)+w_{c}(0),
\end{aligned}
$$

where the first inequality follows from the fact that the buyer can buy 1 unit of money in the centralized meeting, the second inequality from the second inequality in (11), and the equality from (1). Following the same logic, we have

$$
\begin{aligned}
-c(y-\epsilon)+w_{c}(2-\eta) & \geq-c(y-\epsilon)+z(1-\eta)+\delta w_{p}(1)> \\
-c(y)+z+\delta w_{p}(1) & =-c(y)+w_{c}(2) .
\end{aligned}
$$

Therefore, by (12) and (13) the offer $(y-\epsilon, 1-\eta)$ Pareto dominates $(y, 1)$, the planner's proposal, a contradiction.

Sufficiency. Consider any $\tilde{y} \in\left(0, \min \left\{y_{\max }, y^{*}\right\}\right]$ and let $\tilde{z}$ be such that $(\tilde{y}, \tilde{z}) \in V$. Our candidate for the completion of the planner's proposal is

$$
g\left(m, m^{\prime}\right)=\arg \max _{(y, x) \in \mathbb{R}_{+} \times[0, m]}[-c(y)+\tilde{z} x]
$$

subject to

$$
u(y)-\tilde{z} x \geq\left(\mathbb{I}_{m \geq 1}\right)(u(\tilde{y})-\tilde{z})+\left(\mathbb{I}_{m<1}\right) u(0),
$$

where $\mathbb{I}$ is the indicator function. The constraint set in this problem is not empty and the solution exists and is unique; moreover, the displayed constraint holds at equality at the solution. (Notice that the problem has the form of a Pareto problem and that the period return to a buyer with $m \neq 1$, a defector, is minimized subject to a lower bound. The lower bound for $m>1$ is that implied by an output trade equal to $\tilde{y}$, and a transfer of 
1 unit of money from the buyer to the seller; the lower bound for $m<1$ is that implied by no trade. The objective is the period return of the seller.)

We first show that $g\left(1, m^{\prime}\right)=(\tilde{y}, 1)$. If $x=1$, then constraint (14) at equality implies $y=\tilde{y}$. Otherwise, the constraint on the trade of money is not binding. Then, after substituting $\tilde{z} x$ from (14) at equality into the objective, we see that $y$ is chosen to maximize $u(y)-c(y)$. Hence, $y=y^{*}$. But, because $\tilde{y} \leq y^{*}$ and $x<1$, this means that constraint (14) is slack, and then the value of the objective can be increased by increasing $m$, a contradiction. Thus $g\left(1, m^{\prime}\right)=(\tilde{y}, 1)$.

Next, we propose equilibrium strategies and beliefs. The stage- 1 strategies are dictated by the requirements of a simple equilibrium: agents are truthful, they say yes to any proposal, and the buyer proposes the planner's proposal. The strategy in the centralized meeting, $s_{c}$, is such that any agent leaves with 1 unit of money. For beliefs, we assume that reports are believed. Notice that Bayes rule only applies to reports that say that 1 unit of money is held.

Given the proposals and the strategies, we can calculate the value functions. It follows that $w_{c}(m)$ is given by $(8)$ and that $w_{p}(m)$ is given by $(9)$, where the latter is a consequence of the binding constraint in the problem that defines $g$. (That is, without knowing the trades that solve the above problem for $m \neq 1$, the binding constraint gives us the payoffs needed to specify the value functions, which are those in the proof of proposition 1.)

Now we confirm that the strategies are optimal. ${ }^{10}$ Because $s_{c}$ and the value functions are those in Proposition 1, it follows from the proof of that proposition that $s_{c}$ is optimal. As regards the strategies for pairwise meetings, if both agents are truthful and say yes, then the planner's proposal is an optimal proposal by the buyer because of the Pareto form of the problem that defines $g$ and the lower bound in (14). And, because the value functions are those of Proposition 1, it is optimal for both agents to say yes. It remains to show that agents tell the truth.

Consider first a buyer with $m^{\prime} \geq 1$ who makes an announcement $m<m^{\prime}$ and with respective solutions $\left(y^{\prime}, x^{\prime}\right)$ and $(y, x)$. Also, let $\Delta=m^{\prime}-m$. If

\footnotetext{
${ }^{10}$ Again, the principle of one-period deviations can be invoked (see the last footnote).
} 
$m \geq 1$, then the payoff to misrepresentation is

$$
\begin{aligned}
u(y)+w_{c}\left(m^{\prime}-x\right)= & u(y)+w_{c}(m-x)+z \Delta= \\
u(\tilde{y})+w_{c}(m-1)+z \Delta= & u(\tilde{y})+w_{c}\left(m^{\prime}-1\right)= \\
& u\left(y^{\prime}\right)+w_{c}\left(x^{\prime}-m^{\prime}\right),
\end{aligned}
$$

where the first and third equalities follow from the fact that $w_{c}$ is affine, and the second and fourth from constraint (14) at equality. If, instead, $m<1$, then the payoff to misrepresentation is

$$
\begin{aligned}
& u(y)+w_{c}\left(m^{\prime}-x\right)=u(0)+w_{c}\left(m^{\prime}\right)< \\
& u(\tilde{y})+w_{c}\left(m^{\prime}-1\right)=u\left(y^{\prime}\right)+w_{c}\left(m^{\prime}-x^{\prime}\right),
\end{aligned}
$$

where the equalities follow from (14) at equality, and the inequality follows from the second inequality that defines $V$.

Next, consider a buyer $m^{\prime}<1$. Then the payoff to misrepresentation is

$$
u(y)+w_{c}\left(m^{\prime}-x\right)=u(0)+w_{c}\left(m^{\prime}\right)=u\left(y^{\prime}\right)+w_{c}\left(m^{\prime}-x^{\prime}\right),
$$

where both equalities follow from (14) at equality.

Finally, because the solution for $g$ does not depend on the seller's announcement, the seller cannot gain by making a false announcement. This completes the proof of sufficiency except for $\tilde{y}=0$, which is obviously CP implementable.

This characterization implies our claim that if people are sufficiently patient, then the first best is CP implementable. If they are, then as noted above $y^{*} \leq y_{\max }$, and we can CP implement $y^{*}$.

It may seem surprising that we can implement more allocations than can be achieved under generalized Nash bargaining. In a static model, the set of all such generalized Nash bargaining outcomes is the set of coalition-proof allocations taking as given the agent endowments. Here, we can choose how to divide the gains from trade as a function of the endowments, the money holdings brought into the meeting. That additional freedom allows us to implement more stationary allocations. ${ }^{11}$

\footnotetext{
${ }^{11}$ See Zhu and Wallace 2007 for another application of such freedom.
} 
As we claimed, all of the above results carry over to the version with quasilinear stage-2 preferences, provided that they are consistent with a degenerate distribution of money. If they are, then quasi-linear stage- 2 preferences imply an affine $w_{c}$ function that differs from the one above only by having a different constant - a constant that plays no role in any of the arguments above.

Finally, it is evident from our proofs that the ability of agents to hide money does not restrict the set of IR or CP implementable allocations. For CP implementability, this is due to the linearity (or quasi-linearity) of stage2 preferences, as can be seen in the proof of proposition 2 . We should not expect the same results to hold in more general models.

\section{Deflation and inflation}

A standard exercise in the Lagos-Wright model is to study the effects of lump-sum taxes, positive or negative, levied in the centralized meeting, stage 2 , and used to finance interest on money, positive or negative. And, it is standard to make the payment of that tax mandatory, not subject to any no-commitment restriction. ${ }^{12}$ Here we take no-commitment into account by making participation in the centralized meeting voluntary. In particular, any agent can choose not to participate in the centralized meeting, while continuing on into the next pairwise meeting. Because trading histories are private, a person cannot be punished in the future for skipping the centralized meeting.

Taxes are introduced in the following way. Someone who enters the centralized meeting with $m$ units of money receives $\tau$ units of money as a lumpsum transfer (where $\tau$ may be negative), and pays a tax such that after-tax money holdings are given by $\frac{m+\tau}{1+\tau}$. This is equivalent to letting the money supply grow at rate $\tau$ and normalizing all nominal quantities and prices by the per capita stock of money. In particular, if the per capita nominal stock of money satisfies $M_{t+1}=(1+\tau) M_{t}$ and $m^{\prime}$ denotes an individual's nonnormalized holding entering stage 2 , then the post-transfer holding as a fraction of $M_{t+1}$ is $\frac{m^{\prime}+\tau M_{t}}{M_{t+1}}=\frac{m+\tau}{1+\tau}$, where $m=\frac{m^{\prime}}{M_{t}}$. The nonnormalized price of money at $t$, denoted $p_{t}^{\prime}$, satisfies $p_{t}^{\prime}=\frac{p}{(1+\tau)^{t}}$. Therefore, the rate of return on money is $\frac{p_{t+1}^{\prime}}{p_{t}^{\prime}}-1=-\frac{\tau}{1+\tau}$. The Friedman-rule is $\tau=\delta-1$. In order to avoid the

\footnotetext{
${ }^{12}$ Since first formulating the material in this section, we have come across Andolfatto (2008), which also departs from mandatory taxation.
} 
well-known indeterminacy at the Friedman rule, we study $\tau>\delta-1$.

We begin with necessary conditions for IR implementability, the analogue of lemma 1.

Lemma 2 If $\tau>\delta-1$ and $(y, z)$ is IR implementable, then $g(1,1)=(y, 1)$, $p=(1+\tau) z$, and

$$
w_{p}^{t}(1)=h(y) \text { and } w_{c}^{t}(m)=z(m-1)+\delta w_{p}^{t}(1) \text { for } m \in\{0,1,2\} .
$$

Proof. As in the proof of lemma 1, there is a simple IR equilibrium consistent with $(y, z)$. The bound on $\tau$ implies that excess money is not carried from one stage 2 to the next stage 2 . That implies $g(1,1)=(y, 1)$, which, in turn, implies that money holdings are in the set $\{0,1,2\}$ at the beginning of stage 2 . And, in order that consumption at stage 2 be consistent with the allocation $(y, z)$ and that each person leave stage 2 with 1 unit of money, we must have

$$
p\left(\frac{m+\tau}{1+\tau}-1\right)=\left\{\begin{array}{l}
-z \text { if } m=0 \\
0 \text { if } m=1 \\
z \text { if } m=2
\end{array} .\right.
$$

It follows that $p=(1+\tau) z$. The value-function conclusions in (15) follow.

Although this result does not cover the Friedman-rule rate-of-return, that is not a concern. The usual treatment of that case is to select the $g(1,1)=$ $(y, 1)$ simple equilibrium from among many such equilibria for that case. Given that selection, our proof extends to the Friedman-rule rate-of-return.

Because the games have to be amended in slightly different ways for the deflation and inflation cases, we study those cases separately.

\subsection{Deflation}

In this case, we present one result: if $(y, z)$ is IR implementable with deflation, then it is IR implementable with no deflation, with $\tau=0$. This result and proposition 2 imply that deflation is useless. Because the result pertains to IR implementability, we here describe only how to amend that definition to permit consideration of a tax.

Planner proposal: a fourth object, $\tau \in \mathbb{R}_{-}$, is added to what constitutes a planner's proposal in definition 1. 
IR game: The part of the game for stage 1 is unchanged. At the conclusion of stage 1 , each person chooses from $\{y e s, n o\}$, where yes means proceeding to stage 2 , the centralized meeting, and no means skipping it. If yes is played, then the person is subject to the taxes implied by $\tau$ and, as in the no-tax game, chooses a budget-feasible trade at the price in the planner proposal.

It may seem odd to tie paying the lump-sum tax to receiving a return on money. After all, if there were actual deflation, then a person could conceivably not pay the lump-sum tax, but still receive the return on money implied by deflation. However, that kind of defection, which we do not permit, would only further restrict what can be implemented.

$I R$ strategy. This is unchanged except that we replace $s_{c}^{t}$, the stage 2 strategy, by $s_{c}^{\prime t}=\left(s_{c 1}^{\prime t}, s_{c 2}^{\prime t}\right)$, where $s_{c 1}^{\prime t}: \mathbb{M} \rightarrow\{$ yes, no $\}$ (the stage-2 participation choice) and $s_{c 2}^{\prime t}$ is defined as was $s_{c}^{t}$ with the budget implied by the tax scheme.

The definitions of equilibrium, simple equilibrium, and IR implementability are not affected. Now we can prove that deflation does not help.

Proposition 3 If $\tau \in(\delta-1,0]$ and $(y, z)$ is IR implementable, then $(y, z) \in$ $V$.

Proof. Suppose that $(y, z)$ is IR implementable. Someone who enters a pairwise meeting without money has the following option: with probability $1 / N$ produce, acquire a unit of money, and enter the centralized meeting; otherwise skip the centralized meeting. Therefore,

$$
w_{p}(0) \geq \frac{-c(y)+w_{c}(1)}{N}+\frac{N-1}{N} \delta w_{p}(0),
$$

or, equivalently,

$$
w_{p}(0) \geq \frac{-c(y)+w_{c}(1)}{N-(N-1) \delta}=\frac{-c(y)+\delta h(y)}{N-(N-1) \delta},
$$

where the equality uses (15) (see lemma 2). But for IR implementability, the payoff for someone who enters stage 2 without money and participates in the centralized meeting, $w_{c}(0)$ as given by (15), must exceed that implied by skipping the centralized meeting, $\delta w_{p}(0)$. By $(17)$, that requires

$$
-z+\delta h(y) \geq \delta \frac{-c(y)+\delta h(y)}{N-(N-1) \delta} .
$$


This is easily seen to be equivalent to the second inequality that defines $V$.

And, because a seller with 1 unit of money in a pairwise meeting has no trade as an option, we require $-c(y)+w_{c}(2) \geq w_{c}(1)$. According to (15), this is the first inequality that defines $V$.

In principle, deflation can be beneficial because it transfers resources from those without money to those who have money - that is, from people who were consumers in pairwise meetings to people who were producers. When participation constraints are binding, in the sense that $y^{*}>y_{\max }$, everyone would be willing to commit to $y^{*}$ before going to pairwise meetings, if commitment were possible. But this means taking a gamble that involves a net loss for someone who turns out to be a producer, and a gain for someone who is a consumer, such that the expected gain outweighs the expected loss. When commitment is not possible, the producer would balk, because $c\left(y^{*}\right)<R u\left(y^{*}\right)$, and the highest feasible output is then $y_{\max }$, with $c\left(y_{\max }\right)=z=R u\left(y_{\max }\right)$. If it were possible to enhance the value of money in such a way as to retrieve some of the consumer's gain in the pairwise meeting and transfer it to the producer, then the participation constraint could be relaxed. But when this constraint is binding, the consumer is indifferent between skipping the centralized meeting and exchanging $z$ for a unit of money in that meeting. Thus, any scheme that taxes the consumer for participation in the centralized meeting is not feasible unless the tax can be made mandatory. Indeed, no mattter what form of taxation might be used, any such scheme implies some value of $z$ - to be produced by those who were consumers in pairwise meetings and to be consumed by those who were producers. Thus, the value functions are those given by Lemma 2, and Proposition 3 applies. Therefore, when $y^{*}>y_{\max }$, there is no feasible intervention such that $y>y_{\max }$ unless participation in the centralized meeting is mandatory.

Because we tie avoiding the tax to skipping the centralized meeting, Proposition 3 uses linearity of preferences in the centralized meeting, rather than quasi-linearity, in an essential way. But quasi-linearity does not, of course, justify making the tax mandatory. Instead, it requires a more detailed formulation of tax enforcement and how it is related to other activities in the centralized meeting. 


\subsection{Inflation}

It is also standard to use versions of the Lagos-Wright model to provide a positive analysis of the welfare costs of inflation. In order to have our tax transfer scheme for $\tau>0$ correspond to inflation, which holders of money cannot avoid (except by holding less money), we here make stage- 2 participation mandatory, in the sense that any money held by someone who skips the centralized meeting becomes worthless. With that innocuous change, our formulation is equivalent to the usual formulation with inflation.

To use the model to study the welfare effects of inflation, we characterize the set of IR and CP implementable allocations taking $\tau \in \mathbb{R}_{+}$as given. As we will see, the lump-sum transfer tends to reduce the set of IR implementable allocations because it tempts people to leave the centralized meeting without money in order to avoid the inflation tax.

We show that for $\tau>0$, the set of IR implementable allocations is

$$
V(\tau)=\{(y, z): c(y) \leq z \leq R(\tau) u(y)\} .
$$

where

$$
R(\tau)=\frac{\delta}{N(1+\tau)-(N-1) \delta} .
$$

Notice that $(y, z) \in V(\tau)$ if and only if $y \in\left[0, y_{\max }(\tau)\right]$, where $y_{\max }(\tau)$ is the unique positive solution for $y$ to $c(y)=R(\tau) u(y)$, with $R(0)=R$ and $y_{\max }(0)=y_{\max }$ in terms of the notation used above. Clearly, $R(\tau)$ is strictly decreasing in $\tau$ and $R(\tau) \rightarrow 0$ as $\tau \rightarrow \infty$. It follows that $y_{\max }(\tau)$ is strictly decreasing in $\tau$ and that $y_{\max }(\tau) \rightarrow 0$ as $\tau \rightarrow \infty$. After we prove the claim about IR implementable allocations, conclusions about CP implementable allocations will follow immediately from the argument used to prove Proposition 2.

The proof of the next proposition is almost identical to that of proposition 1.

Proposition 4 Given $\tau \in \mathbb{R}_{+}$, there exists z such that $(y, z)$ is IR implementable if and only if $y \in\left[0, y_{\max }(\tau)\right]$.

Proof. Necessity. At stage 1, defection to no-trade by a seller with 1 unit of money assures a payoff no less than $w_{c}(1)$, while following $g(1,1)=(y, 1)$ gives the seller $-c(y)+w_{c}(2)$. Thus, it must be the case that

$$
c(y) \leq w_{c}(2)-w_{c}(1)=z,
$$


where the equality follows from (15) (see lemma 2). This is the first inequality that defines $V(\tau)$.

Next, consider an agent who enters stage 2 with 0 money. A feasible alternative to $w_{c}(0)$ is to leave stage 2 without money and to resume feasible equilibrium actions starting at the next date. That such a deviation not be undertaken requires

$$
-z+\delta w_{p}(1) \geq \tau z-\frac{\delta c(y)}{N}-\frac{(N-1) \delta z}{N}+\delta^{2} w_{p}(1)
$$

where the left side is the no-deviation payoff and the right side is the deviation payoff including the payoff from consuming the value of the lump-sum transfer, $\frac{p \tau}{1+\tau}=z \tau$. By (15), (22) is equivalent to

$$
\delta(1-\delta) h(y) \geq \tau z+\left(1-\delta \frac{N-1}{N}\right) z-\frac{\delta c(y)}{N} .
$$

Using the definition of $h(y)$, it is easily seen that this is the second inequality that defines $V(\tau)$.

Sufficiency. Our candidates for $g(1, m)$ and $g(m, 1)$ are the same as in the proof of proposition 1 (see (7)). And, again, we propose that an equilibrium strategy has $s_{c}(m)$ (consumption at stage 2) such that each person leaves stage 2 with 1 unit of money. If the above trades are carried out, then the initial degenerate distribution persists and the implied value functions are given by the expressions in the proof of proposition 1 (see (8) and (9)). To complete the proof, we have to show that these induce truth-telling and playing yes at stage 1 and also induce the proposed stage-2 strategy.

Given $g$ in (7), truthfulness is a weakly dominant strategy for everyone. Now we show that a buyer with $m \geq 1$ says yes and that any seller says yes. For the former, we need to show that $u(y)+w_{c}(m-1) \geq w_{c}(m)$ or by $(8)$ that $u(y) \geq z$. This follows from the second inequality that defines $V(\tau)$. For the latter, we need to show that $-c(y)+w_{c}(m+1) \geq w_{c}(m)$, which is just the first inequality that defines $V(\tau)$.

Now we show that the stage- 2 proposed strategy, $s_{c}(m)=z(m-1)$, is optimal for agents. We proceed by considering two exhaustive sets of alternatives.

Case (i): $s_{c}(m)<z(m-1)$. This implies leaving stage 2 with more than 1 unit of money. However, just as in the proof of lemma 1, this implies carrying some money from the current stage 2 to the next stage 2 , which is not optimal. 
Case (ii): $s_{c}(m)>z(m-1)$. This implies leaving stage 2 with less than 1 unit of money. But, any amount of money less than one unit is not spent in a pairwise meeting. Therefore, it is better to leave stage 2 with 0 . That implies

$$
s_{c}(m)=p\left(\frac{m+\tau}{1+\tau}\right)=z(m+\tau) .
$$

According to (9), the implied payoff is

$$
\left.z(m+\tau)+\delta\left[\frac{-c(y)}{N}-z \frac{(N-1)}{N}\right)+\delta h(y)\right]
$$

We have to show that this is weakly dominated by the payoff from choosing $s_{c}(m)=z(m-1)$, which according to $(9)$ is $z(m-1)+\delta h(y)$. The required inequality is equivalent to

$$
\left.z(1+\tau)+\delta\left[\frac{-c(y)}{N}-z \frac{(N-1)}{N}\right)+\delta h(y)\right] \leq \delta h(y)
$$

which, in turn, is equivalent to inequality (23). But, as noted above, inequality (23) is equivalent to the second inequality that defines $V(\tau)$.

Now we turn to CP implementability. There is an analogue of proposition 2 with $y_{\max }(\tau)$ in place of $y_{\max }$.

Corollary 1 Given $\tau \in \mathbb{R}_{+}$, there exists $z$ such that $(y, z)$ is CP implementable if and only if $y \in\left[0, \min \left\{y^{*}, y_{\max }(\tau)\right\}\right]$.

The proof of the corollary follows exactly the logic of the proof of proposition 2 except that $y_{\max }(\tau)$ replaces $y_{\max }$. With this corollary in hand, we can describe the implied welfare cost of inflation and how that cost compares to those for given trading protocols.

In this model, it is reasonable to measure the welfare cost of inflation by some increasing function of $y^{* *}-y$, where $y^{* *}=\min \left\{y^{*}, y_{\max }(0)\right\}$ and $y$ is stage 1 output. If $y$ is chosen optimally from the CP implementable set, then $y=\min \left\{y^{*}, y_{\max }(\tau)\right\}$ and the welfare cost of inflation is determined by $y^{* *}-\min \left\{y^{*}, y_{\max }(\tau)\right\} .{ }^{13}$ All the trading protocols that have been studied give rise to IR implementable allocations and to $y<y^{* *}$. It follows that

\footnotetext{
${ }^{13}$ If there were a revenue need that could only be met through inflation, then the planner would want to choose the best CP implementable allocation.
} 
the implied stage-1 output found for a given trading protocol satisfies $y \leq$ $\min \left\{y^{*}, y_{\max }(\tau)\right\}$. Therefore, as should be no surprise, the welfare cost we find is no greater than that found for any given protocol.

Lagos and Wright argue that if the inflation rate is slightly above the Friedman-rule rate, and if the trading protocol is that buyers make take-itor-leave-it offers to sellers, then the cost of inflation is small, by the envelope theorem. Our result is quite different (and it has nothing to do with the Friedman-rule). First, if $y^{* *}=y^{*}$, then inflation has no cost, up to the point where $y^{*}=y_{\max }(\tau)$. Second, if $y^{* *}=y_{\max }(0)$, meaning that the first-best level of output cannot be achieved because the seller's participation constraint is binding, then inflation has a first-order cost, because it exacerbates this constraint.

Our result can be illustrated by reconsidering the cost of inflation in the parameterized model discussed by Lagos and Wright (2005). The cost and utility functions are given by $c(y)=y$ and $u(y)=\frac{y^{1-\eta}}{1-\eta}$ with $\eta=\frac{3}{10}$, the discount factor is $\delta=\frac{1}{1.04}$, and the probability of a single-coincidence meeting is $\frac{1}{N}=\frac{1}{2}$. For this model, $y^{*}=1$ and $y_{\max }(\tau)=\left(\frac{R(\tau)}{1-\eta}\right)^{\frac{1}{\eta}}$. In the case of the Nash bargaining protocol, Lagos and Wright obtain $y=.442$ when the inflation rate is zero, and $y=.143$ when the inflation rate is $10 \%$, implying a large welfare cost. But for these parameter values we have $y_{\max }(0.1)=1.41$, so that the first-best allocation is in the $\mathrm{CP}$ implementable set; in fact the welfare cost is zero for inflation rates below $16.7 \%$.

\section{Concluding remarks}

Our results imply that entire classes of trading protocols that have been studied are missing good coalition-proof implementable allocations. There is no reason to think that that result is limited to the Lagos-Wright model. Thus, much hinges on whether we study all such allocations or choose a particular class of trading protocols. Existing work also overstates the welfare cost of inflation and the beneficial role of tax schemes used to finance the payment of interest on money. It overstates the beneficial role of such tax schemes for two reasons. First, that work understates what can be achieved without such tax schemes. Second, most of it fails to subject tax schemes to restrictions like no-commitment and imperfect monitoring, the restrictions that give money a role. 


\section{References}

[1] Andolfatto, David. 2008. "The Simple Analytics of Money and Credit in a Quasi-linear Environment." Working paper.

[2] Deviatov, Alexei. 2006. "Money Creation in a Random Matching Model." Topics in Macroeconomics 6 (3), article 5.

[3] Kocherlakota, Narayana. 1998. "Money is Memory." J. of Econ. Theory 81 (2): 232-51.

[4] Lagos, Ricardo and Guillaume Rocheteau. 2005. "Inflation, Output, and Welfare," International Econ. Rev. 46 (2): 495-522.

[5] Lagos, Ricardo, and Randall Wright. 2005. "A Unified Framework for Monetary Theory and Policy Analysis, J. of Political Economy 113 (3): 463-84.

[6] Rocheteau, Guillaume, and Randall Wright. 1995. "Money in Search Equilibrium, in Competitive Equilibrium, and in Competitive Search Equilibrium," Econometrica 73 (1): 175-202.

[7] Shubik, Martin. 1973. "Commodity Money, Oligopoly, Credit and Bankruptcy in a General Equilibrium Model, Western Economic Journal, 11 (1), 24-38.

[8] Wallace, Neil, and Tao Zhu. 2007. "Float on a Note." J. of Monetary Econ. 54 (2):229-46.

[9] Zhu, Tao. 2008. "Equilibrium Concepts in the Large-Household Model." Theoretical Economics 3 (2) 257-81.

[10] Zhu, Tao and Neil Wallace. 2007. "Pairwise Trade and Coexistence of Money and Higher-Return Assets." J. of Econ Theory, 133 (1) 524-35. 